\title{
MATHEMATICAL MODELLING OF THE EVOLUTION OF HUMAN BEHAVIOURS AND STRATEGIC CHOICE
}

\author{
SARA L. LOO
}

(Received 31 July 2019; first published online 13 September 2019)

2010 Mathematics subject classification: primary 91D15; secondary 92D10, 92D25, 92D50.

Keywords and phrases: evolutionary anthropology, male strategies, cultural evolution, pair bonding, paternal care.

Evolutionary anthropology seeks answers to the eternal philosophical question of who we are and how we have come to be the way that we are. Studies within evolutionary anthropology attempt to explain the evolution of uniquely human behaviours by understanding the mechanisms and trade-offs that drive different evolutionary trajectories.

There are clear differences between humans and our closest cousins, the great apes-including our long post-menopausal life span, pair bonding and large-game hunting behaviours. But the mechanisms and trade-offs that drove human behaviour apart from other great apes remain largely unquantified.

This thesis attempts to fill in some of these gaps of knowledge by considering male strategic choice. Hypotheses from evolutionary anthropology are explored and dynamic mathematical models built to investigate pay-offs to different strategies and key parameters that define divergent evolutionary pathways. The evolutionary equilibria of these dynamic models at great ape-like life histories are compared to that of hunter-gatherer-like life histories in order to shed light on these driving mechanisms.

One investigation considers the evolution of pair bonding in humans. Models of male reproductive strategies are developed and analysed to demonstrate the promise of mate guarding as a pathway to social monogamy in humans. Mate guarding outperforms strategies of paternal care in hunter-gatherer-like male-biased sex ratios, while multiple mating is the evolutionary equilibrium at chimpanzee-like femalebiased sex ratios. This not only shows care to be an insufficient pathway to social monogamy, but also links male reproductive strategies to the grandmother hypothesis through dependency on sex ratios. Here, where pay-offs are received in increased

Thesis submitted to the University of Sydney in September 2018; degree awarded on 25 March 2019; supervisor Peter Kim.

(C) 2019 Australian Mathematical Publishing Association Inc. 
relative likelihood of obtaining paternities, mate guarding outperforms other strategies as mates become scarce.

Male competition is then investigated more generally by comparing strategies of competition that increase the likelihood of obtaining paternities, as with mate guarding, with strategies of care that pay off in differential offspring survivorship. The ordinary differential equation model developed explores the strength of selection on traits that increase pay-offs to each strategy. This model explores the question of sexrole divergence, which outlines differences in post-mating behaviours where males tend towards competition while females more often tend towards care. This question of competition versus care has been pervasive in the anthropological literature. The model provided shows the clear dependence of strategic inheritance on life history parameters, and highlights regions where competition outperforms care in a range of increasingly realistic scenarios.

A further example is then presented to demonstrate the counter-intuitive coevolution of low fertility at high socioeconomic status. This applies ideas of Darwinian selection to a problem of cultural evolution in a contemporary society. In the integro-difference equation model developed, cultural evolution is used as a framework within which to consider the effect of nonparental imitation.

This thesis concludes that competition between males is highly significant in the evolution of reproductive strategies. While traits that increase competition in males may manifest in a variety of forms, such as mate guarding and signalling through large-game hunting as modelled here, the studies included in this thesis demonstrate that at hunter-gatherer-like parameters, greater pay-offs are more often obtained through investment in increased relative likelihood of paternities rather than differential offspring survivorship. In addition to this general observation, the studies show that coexistence or persistence of a noncompetitive strategy can also occur, given specifically defined life history parameters or parameters of benefit.

Uncovering significant parameters that drive the evolution of different male strategies is influential in not only guiding further ethnographic research but also providing reasons as to why we are so different from other great apes, and other mammals in general.

Some of this research has been published in $[1,2]$.

\section{References}

[1] S. L. Loo, M. H. Chan, K. Hawkes and P. S. Kim, 'Further mathematical modelling of mating sex ratios and male strategies with special relevance to human life history', Bull. Math. Biol. 79(8) (2017), 1907-1922.

[2] S. L. Loo, K. Hawkes and P. S. Kim, 'Evolution of male strategies with sex-ratio-dependent payoffs: connecting pair bonds with grandmothering', Philos. Trans. R. Soc. Lond. B 372(1729) (2017), 20170041.

SARA L. LOO, School of Mathematics and Statistics,

University of Sydney, NSW 2006, Australia

e-mail: sara.loo@sydney.edu.au 